\title{
P I 2-09. Immune responses in controlled and uncontrolled HIV infection to a designed HIV immunogen sequence focused on conserved regions of the viral genome
}

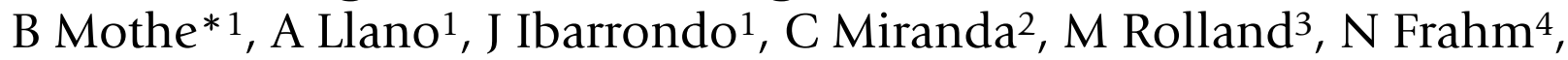
A Khatri ${ }^{5}$, D Heckerman ${ }^{6}$, J Mullins ${ }^{3}$ and C Brander ${ }^{1}$

\author{
Address: ${ }^{1}$ Hospital Germans Trias i Pujol, IrsiCaixa AIDS Research Institute-HIVACAT, Badalona, Spain, ${ }^{2}$ Lluita contra la Sida' Foundation, \\ Hospital Germans Trias i Pujol, Badalona, Spain, ${ }^{3}$ Department of Microbiology. University of Washington, Washigton, USA, ${ }^{4}$ Fred Hutchinson \\ Cancer Research Center, Seattle, WA, USA, 5 Partners AIDS Research Center, Massachusetts General Hospital, Boston, MA, USA and ${ }^{6}$ Microsoft \\ Research, Redmond, CA, USA \\ * Corresponding author
}

from AIDS Vaccine 2009

Paris, France. 19-22 October 2009

Published: 22 October 2009

Retrovirology 2009, 6(Suppl 3):PI75 doi:10.1 I86/I742-4690-6-S3-PI75

This abstract is available from: http://www.retrovirology.com/content/6/S3/PI75

(C) 2009 Mothe et al; licensee BioMed Central Ltd.

\section{Background}

Inclusion of conserved regions of the HIV genome into vaccine candidates may help to overcome problems of global sequence diversity and could provide beneficial effects by inducing $\mathrm{T}$ cell responses for which CTL escape is likely associated with fitness costs.

\section{Methods}

We rationally designed an immunogen sequence consisting of "conserved elements" (CE) that includes 7 segments of the Gag p24 protein. Responses to CE and to the rest of p24 and the entire viral proteome were assessed in HIV controllers $(n=15)$ and HIV non-controllers $(n=10)$ using a comprehensive 18 mer overlapping peptide set spanning the entire viral proteome and an 11 mer overlapping (by 10 residues) peptide set spanning Gag p24 and covering all $\mathrm{CE}$ and the most frequent occurring mutations in this region.

\section{Results}

Controllers and non-controllers mounted comparable number of responses to either entire p24 or CE only ( 8 vs. 7 for p24 and 3 in both groups for CE). Despite these frequent reactivities in non-controllers, the contribution of CE responses to total p24 magnitude was significantly lower in non-controllers ( $\mathrm{p}=0.043)$. In addition, control- lers showed a significantly higher recognition of variants, both to the CE (median variant reactivity 3 vs $1, \mathrm{p}=0.01$ ) and total p24 (5 vs 2.5, p =0.01), 44\% of which were responses to variants only, without reacting to the consensus sequence. Importantly, the observed responses were not driven by CTL activity to epitopes restricted by HLA B27, HLA B57 or HLA B58, as such subjects were previously excluded.

\section{Conclusion}

Cross-reactive responses able to recognize CE epitope variants present in controllers could possibly explain their superior ability to control in vivo HIV replication. In addition, specific responses to subdominant sequence variants that were not elicited by consensus sequences may represent more immunogenic viral sequences that could provide candidates for the induction of responses able to protect from heterologous viral challenge. 